\title{
A single institution analysis of low-dose-rate brachytherapy: 5-year reported survival and late toxicity outcomes
}

\author{
Michael Chao, AFRACMA, FRANZCR', Sandra Spencer, Dip App Sci Med Rad', Mario Guerrieri, FRANZCRl', \\ Wei Ding, MD, MScl, Mehran Goharian, BSc, MSc, PhD!, Huong Ho, BSc, MScl, Michael Ng, FRANZCR!, \\ Danielle Healey, BSc, Grad Dip Couns', Alwin Tan, MBBS, FRACS², Chee Cham, MBBS, FRACS, FRCS², \\ Daryl Lim Joon, MBBS, FRANZCR ${ }^{3}$, Nathan Lawrentschuk, MBBS, PhD, FRACS 3 , Douglas Travis, MBBS, FRACS ${ }^{4}$, \\ Shomik Sengupta, MS, MD, FRACS 3 , Yee Chan, FRACS 3 , Andrew Troy, FRACS 3 , Trung Pham, MBBS, FRACS 5 , \\ David Clarke, FRACS ${ }^{4}$, Peter Liodakis, FRACS 3 , Damien Bolton, MD, MBBS, BA, FRACS, FRCS ${ }^{3}$ \\ 'Genesis Cancer Care Victoria, Melbourne, ${ }^{2}$ The Bays Hospital, Mornington, ${ }^{3}$ Austin Health, Heidelberg, ${ }^{4}$ Western Private Hospital, Footscray. \\ ${ }^{5}$ The Valley Private Hospital, Mulgrave, Australia
}

\begin{abstract}
Purpose: To report the 5-year biochemical relapse-free survival (BRFS), overall survival (OS), and long-term toxicity outcomes of patients treated with low-dose-rate (LDR) brachytherapy as monotherapy for low-to intermediate-risk prostate cancer.

Material and methods: Between 2004 and 2011,371 patients were treated with LDR brachytherapy as monotherapy. Of these, 102 patients (27\%) underwent transurethral resection of the prostate (TURP) prior to implantation. Follow-up was performed every 3 months for 12 months, then every 6 months over 4 years and included prostate specific antigen evaluation. The biochemical relapse-free survival (BRFS) was defined according to the Phoenix criteria. Acute and late toxicities were documented using the Common Terminology Criteria for Adverse Events version 4.0. The BRFS and OS estimates were calculated using Kaplan-Meier plots. Univariate and multivariate analyses were performed to evaluate outcomes by pre-treatment clinical prognostic factors and radiation dosimetry.

Results: The median follow-up of all patients was 5.45 years. The 5-year BRFS and OS rates were $95 \%$ and $96 \%$, respectively. The BRFS rates for patients with Gleason score (GS) $>7$ and GS $\leq 6$ were $96 \%$ and $91 \%$ respectively $(p=0.06)$. On univariate analysis, T1 and T2 staging, risk-group classification, and prostate volumes had no impact on survival at 5 years $(p>0.1)$. Late grade 2 and 3 genitourinary $(\mathrm{GU})$ toxicities were observed in $10 \%$ and $5 \%$ of patients respectively. Additionally, patients with prior TURP had a greater incidence of late grade 2 or 3 urinary retention $(p=0.001)$. There were 14 deaths in total; however, none were attributed to prostate cancer.

Conclusions: LDR brachytherapy is an effective treatment option in low- to intermediate-risk prostate cancer patients. We observed low biochemical relapse rates and minimal GU toxicities several years after treatment in patients with or without TURP. However, a small risk of urinary retention was observed in some patients.

J Contemp Brachytherapy 2018; 10, 2: 155-161 DOI: https://doi.org/10.5114/jcb.2018.75600
\end{abstract}

Key words: brachytherapy, prostatic neoplasms, transurethral resection of prostate.

\begin{abstract}
Purpose
Low-dose-rate (LDR) brachytherapy has been used in Australia since 1998 as curative treatment, either as a boost to external beam radiotherapy (EBRT) or monotherapy in the management of non-metastatic prostate cancer. More recently, the use of Iodine-125 for permanent LDR brachytherapy implants has gained popularity and received widespread treatment acceptance [1].
\end{abstract}

Several institutions have reported 5- and 10-year biochemical relapse-free survival ranging from $94-96 \%[2,3,4]$ to $87-98.5 \%$ [3,5,6,7], respectively. In addition, a number of reviews have demonstrated that LDR brachytherapy is equally effective as EBRT or radical prostatectomy (RP) alone in patients with newly diagnosed low- or intermediate-risk prostate cancer [8,9,10]; however, Rodrigues et al. [9] suggested that large sample size, well designed ran-
Address for correspondence: Michael Chao, AFRACMA, FRANZCR, Genesis Cancer Care Victoria, Melbourne, Victoria, Australia, Level 5/ 126 Wellington Parade, East Melbourne, Victoria, 3002, Australia, phone: +61 38870 3300, fax: +61 38870 3388, e-mail: michael.chao@genesiscare.com.au
Received: 14.01.2017

Accepted: 13.03.2018

Published: 30.04.2018 
domized control trials, and/or prospective comparative studies were needed.

In spite of these positive findings and recommendations for practice, there has been an overall decline in the use of brachytherapy by the medical and radiation oncology communities. There have been a number of explanations put forward including the current emphasis on less aggressive treatment strategies such as active surveillance to the need for experienced personnel and specialized equipment required for potentially successful brachytherapy outcomes $[11,12]$. Therefore, given its overall decrease in clinical use, the aim of this study was to report on our recent brachytherapy experiences in order to highlight its success as a treatment modality for patients with low- to intermediate-risk prostate cancer, including a cohort which underwent pre-implant trans urethral resection of the prostate (TURP).

\section{Material and methods}

\section{Study setting, design, and ethics}

This prospective case series assessed the effectiveness and safety of permanent LDR brachytherapy for patients with clinically localized prostate cancer at a private radiation oncology center (Radiation Oncology Victoria) in Melbourne, Australia. Approval for outcome analysis was obtained from the board of Radiation Oncology Victoria. Between 2004 and 2011, 371 patients were treated with ${ }^{125} \mathrm{I}$ brachytherapy as monotherapy. All patients underwent a medical history, physical examination, and serum prostate specific antigen (PSA) level. The $\mathrm{T}$ stage was assigned by digital rectal examination. Staging with abdominal/pelvic computerized tomography (CT) and whole-body bone scan was required for patients with Gleason score of 7 . General pathologists in private pathology laboratories assigned biopsy Gleason scores.

Patients were stratified pursuant to D'Amico classification [13]: low-risk: PSA $\leq 10 \mathrm{ng} / \mathrm{ml}$, Gleason score $\leq 6$, and stage cT1-cT2a; intermediate-risk: PSA $10 \mathrm{ng} /$ $\mathrm{ml}$ and Gleason score of 7, and/or stage cT2b prostate cancer. In cases where the T2 sub-stage was not specified but the Gleason score was $<7$ and PSA $<10$, patients were re-classified as "low-risk" rather than be left as "unclassified". The prostate gland size was first assessed on transrectal ultrasound (TRUS) at the time of biopsy and reassessed at volume study regarding feasibility of implant, also taking pubic arch interference and degree of TURP defect, if any, into consideration. If prostate volume exceeded $50 \mathrm{cc}$ but was less than $75 \mathrm{cc}$, patients received a minimum of three months of neoadjuvant androgen deprivation therapy (ADT), either luteinizing hormone-releasing hormone agonist (LHRHa) alone or an anti-androgen such as cyproterone acetate for cytoreduction prior to repeat volume study.

Any patient who underwent a pre-implant TURP had limited resection with only 3-5 grams of tissue removed. If an intravesical median lobe was present, the lobe was resected down to prostatic capsule. In some cases, patients also had a transurethral incision of the prostate
(TUIP). Patients did not proceed to their implant until at least 3 months after their TURP with urodynamic tests and cystoscopy performed to confirm resolution of outlet obstruction and urethral healing.

\section{Planning and treatment procedure}

Volume study was acquired 2 to 4 weeks prior to implant using a Flex Focus 400 scanner (BK Medical Aps, Denmark) and 8848 biplane transducer. All patients underwent bowel preparation with an aperient. Once under general anesthesia, with the patient in standard lithotomy position, a 16F catheter was inserted to identify urethral position. Transverse images were captured from base to apex in $5 \mathrm{~mm}$ intervals, with the urethra standardly aligned to the template along column ' $\mathrm{D}$ ', and imported into the VariSeed (Varian, Palo Alto, CA) treatment planning system (TPS). Pubic arch extent relative to template coordinates was recorded. Technical feasibility of implant in patients who had undergone prior TURP was assessed. The TPS was upgraded from v. 7.0 to v. 8.0 in 2008. The planning target volume (PTV) was created with a $3 \mathrm{~mm}$ expansion anteriorly and 3-5 $\mathrm{mm}$ laterally to the prostate as defined on ultrasound. There was no posterior expansion at the interface of the prostate and anterior rectal wall. Urethra and rectum were contoured as organs at risk. Pubic arch extent was contoured on the widest slice, usually mid gland.

Dosimetry was forward planned utilizing TG-43 formulism as described by the American Association of Physicists in Medicine [14] via modified peripheral loading using the following dose goals and constraints: prescription dose of 145 Gy minimum peripheral dose (MPD) to the PTV; dose to $90 \%$ of the PTV $\left(\mathrm{D}_{90}\right)$ $>120 \%$; volume of PTV receiving prescribed dose $\left(\mathrm{V}_{100}\right)$ $>98 \%$; volume of PTV receiving $150 \%$ of prescribed dose $\left(\mathrm{V}_{150}\right)<65 \%$; volume of PTV receiving $200 \%$ of prescribed dose $\left(\mathrm{V}_{200}\right)<30 \%$; volume of urethra receiving $150 \%$ of prescribed dose $\left(\mathrm{Vu}_{150}\right)<150 \%$ and less than $5 \%$; volume of rectum receiving prescribed dose $\left(\mathrm{Vr}_{100}\right)<1.00 \mathrm{cc}$ and less than $5 \%$. Prior to 2009, needles were manually loaded with seeds at standard $1.0 \mathrm{~cm}$ spacing. During 2009, the transition was made to pre-loaded, customized needles. Median seed strength was $0.423 \mathrm{mCi}$ (IQR: $0.413-0.433$ ) for the majority of cases. For PTV <30 cc, median seed strength was 0.306 mCi (IQR: 0.299-0.312 mCi).

Patients underwent bowel preparation prior to implant. Once under general anesthesia, a 16F catheter was inserted and patient position from volume study was reproduced. A check of the prostate and urinary catheter relative to the template was performed. The prostate was stabilized with an empty needle, which was also used to both establish and recheck the zero-retraction plane after implantation of each row. The implant procedure was performed under TRUS and fluoroscopic guidance. Patients were admitted overnight and discharged the following morning after a successful trial of void. Four weeks post-procedure, all patients attended initial follow-up with the treating radiation oncologist and CT for post-implant dosimetry. 


\section{Evaluation of response: clinical endpoints}

Patients were followed up by the radiation oncologist every three to four months after LDR brachytherapy during the first year. Thereafter, all patients were seen every 6 months over the next 5 years. In addition, patients agreed to PSA testing for a minimum of four years, by which biochemical failure was defined using the Phoenix definition of nadir $+2 \mathrm{ng} / \mathrm{l}$ following implant and excluding any PSA bounce [15].

Relapse-free survival (RFS) was measured from the date of implant to the date of the first failure of any type (biochemical, clinical, metastases or death due to prostate cancer or treatment); and censored for relapse-free patients at the date of their last PSA test or the close-out date, whichever was earlier. Overall survival (OS) time was measured from the date of implant to the date of death from any cause, and censored for those alive at the date of last contact or the closeout date, whichever was earlier.

PSA bounce was defined as a short-term increase of $>0.2 \mathrm{ng} / 1$ in PSA, occurring more than 3 months after implant and followed by a spontaneous decline without intervention [16]. The date of the bounce was recorded at the highest PSA value, and the height of the bounce was measured from the lowest PSA value between implant and bounce. Late gastrointestinal (GI) and genitourinary (GU) toxicities reported 90 days after implantation were graded according to the Common Terminology Criteria for Adverse Events v. 4.0 (CTCAE v. 4). The analysis was based on the evaluation of the maximum toxicity score throughout treatment for each patient.

\section{Statistical analysis}

Descriptive statistics were calculated to characterize the patient, disease, and treatment features as well as toxicities after treatment were presented as mean (plus deviation) or median (interquartile range [IQR]) depending on the underlying distribution of the data. In the case of counts, the crude numbers or percentages were presented. The RFS and OS rates were estimated using the Reverse Kaplan-Meier method. All time to event points were measured from the date of implant to the date of the last PSA test, with censoring considered from the date of relapse. Survival was measured from the date of implant to the date of last contact, with censoring at the date of death. $\mathrm{R}$ statistical software was used for the Kaplan-Meier analyses, whilst StatXact software was used to calculate the 95\% confidence interval (95\% CI) for PSA response.

\section{Results}

\section{Patient characteristics}

Of the 371 patients, 245 (66\%) met the low-risk criteria, whilst 122 (33\%) had intermediate-risk disease. Median age and PSA value at diagnosis were 67 years (IQR: 42-83 years) and $5.6 \mathrm{ng} / \mathrm{ml}$ (IQR: 0.6-15.1 ng/ml), respectively. Median follow-up was 5.45 years with $98 \%$, followed up for at least 4 years. The majority of patients $(73 \%)$ had a Gleason score of $\leq 6$, while $26 \%$ had a Gleason score of 7. Of the 102 patients who underwent pre-implant TURP,
97\% occurred within three months prior to LDR to manage lower urinary tract symptoms. Table 1 shows the details of the patient characteristics.

\section{Dosimetry results}

Implant procedure details and the main dosimetric values are reported in Table 2 . The mean intraoperative prostate volume was 36.7 cc (IQR: 14.7-61.2 cc). An average of 80 implanted seeds (IQR: 46-119) and 23 needles (IQR: 15-38) were used to attain a median prostate $\mathrm{D}_{90}$ of 123.4\% (IQR: 114.3-132.4) and a median prostate $\mathrm{V}_{100}$ of 99.4\% (IQR: 96.4-100\%). Median rectal $\mathrm{RV}_{100}$ was $0.21 \mathrm{cc}$ (IQR: 0-0.96 cc) and 2.99\% (IQR: 0-16.91\%). Post-implant dosimetry performed at day 28-30 demonstrated a median prostate $\mathrm{D}_{90}$ of $99.2 \%$ (IQR: $44.3-148 \%$ ) and a median prostate $\mathrm{V}_{100}$ of $89.5 \%$ (IQR: $60.2-99.7 \%$ ), respectively. Rectal wall was contoured in $92 \%$ of patients. Median post-implant rectal $\mathrm{RV}_{100}$ was $0.87 \mathrm{cc}$ (IQR: 0-3.58 cc) and 2.27\% (IQR: 0-25.77\%) (Table 2).

\section{Time to relapse-free survival}

The biochemical RFS (BRFS) at 5 years using Kaplan-Meier estimates was 95\% (95\% CI: 92-97\%) for all

Table 1. Patient characteristics

\begin{tabular}{|c|c|c|c|}
\hline Characteristics & Variable & $n$ & $\%$ \\
\hline Age at implant (years) & $67(42-83)$ & & \\
\hline No prior TURP, $n=269$ & $\leq 49$ & 6 & 1 \\
\hline \multirow[t]{4}{*}{ Prior TURP, $n=102$} & $50-59$ & 59 & 16 \\
\hline & $60-69$ & 179 & 48 \\
\hline & $70-79$ & 125 & 34 \\
\hline & $\geq 80$ & 3 & 1 \\
\hline \multirow{6}{*}{$\begin{array}{l}\text { T stage (UICC } 7^{\text {th }} \text { ed.) at } \\
\text { presentation }\end{array}$} & $1 c$ & 182 & 49 \\
\hline & $2 a$ & 126 & 34 \\
\hline & $2 b$ & 23 & 6 \\
\hline & $2 c$ & 2 & 1 \\
\hline & 2 & 30 & 8 \\
\hline & Missing & 8 & 2 \\
\hline \multirow[t]{5}{*}{ Gleason score } & 4 & 1 & $<1$ \\
\hline & 5 & 1 & $<1$ \\
\hline & 6 & 266 & 72 \\
\hline & 7 & 98 & 26 \\
\hline & Missing & 5 & 1 \\
\hline \multirow[t]{5}{*}{ PSA at diagnosis $(\mu \mathrm{g} / \mathrm{l})$} & $5.6(0.6-15.1)$ & & \\
\hline & $<4$ & 92 & 25 \\
\hline & $4-10$ & 260 & 70 \\
\hline & $>10$ & 11 & 3 \\
\hline & Missing & 8 & 2 \\
\hline \multirow{3}{*}{$\begin{array}{l}\text { Risk group } \\
\text { (D'Amico classification) }\end{array}$} & Low & 245 & 66 \\
\hline & Intermediate & 122 & 33 \\
\hline & Unknown & 4 & 1 \\
\hline \multirow{3}{*}{$\begin{array}{l}\text { Hormone therapy prior } \\
\text { to LDR brachytherapy }\end{array}$} & No & 286 & 77 \\
\hline & Yes, ADT & 84 & 23 \\
\hline & Yes, non-ADT & 1 & $<1$ \\
\hline
\end{tabular}

Data presented as median (inter-quartile range (IQR)) or number (percentage) $A D T$ - neo-adjuvant hormonal therapy, PSA - prostate-specific antigen, TURP - transurethral resection of the prostate, LDR - low-dose-rate brachyterapy, ADT-adjuvant hormonotherapy 
Table 2. Dosimetry findings

\begin{tabular}{lc} 
Dosimetry characteristics & Median (range) \\
\hline Planning target volume $(c c)$ & $42.7(17.7-68.1)$ \\
\hline Number of seeds implanted $(n)$ & $80(46-119)$ \\
\hline Number of needles $(n)$ & $23(15-38)$ \\
\hline Activity $(\mathrm{U})$ & $0.54(0.38-0.58)$ \\
\hline Prostate $\mathrm{D}_{90}(\%, \mathrm{~Gy})$ & $123.4(114.3-132.4)$ \\
\hline Planned & $179(162-192)$ \\
\hline Delivered & $99.2(44.3-148)$ \\
\hline Prostate $\mathrm{V}_{100}(\%$, Gy) & $143.8(64-215)$ \\
\hline Planned & $99.4(96.4-100)$ \\
\hline Delivered & $144(140-145)$ \\
\hline Rectal wall receiving MPD (cc) & $89.5(60.2-99.7)$ \\
\hline Planned & $129.8(87-145)$ \\
\hline Delivered & $0.21(0-0.96)$ \\
\hline Rectal wall receiving MPD (\%) & $0.87(0-3.58)$ \\
\hline Planned & $2.99(0-16.91)$ \\
\hline Delivered & $2.27(0-25.77)$
\end{tabular}

Data presented as median (inter-quartile range (IQR)) or number (percentage) $A D T$ - neo-adjuvant hormonal therapy, Gy - Gray, MPD - minimum peripheral dose, $D_{90}$ - the minimum dose received by $90 \%$ of the prostate volume, $V_{100}$ - the percent volume of the post-implant prostate receiving $100 \%$ of the prescribed dose

patients. No difference was observed when stratified according to low $(96 \%$ vs. $92 \% ; p=0.090)$. Patients with a Gleason score of 7 had a greater relapse rate than patients with Gleason scores $\leq 6$; however, the findings were not statistically significant $(p=0.063)$ (Figure 1). Similarly, none of the other potential prognostic factors

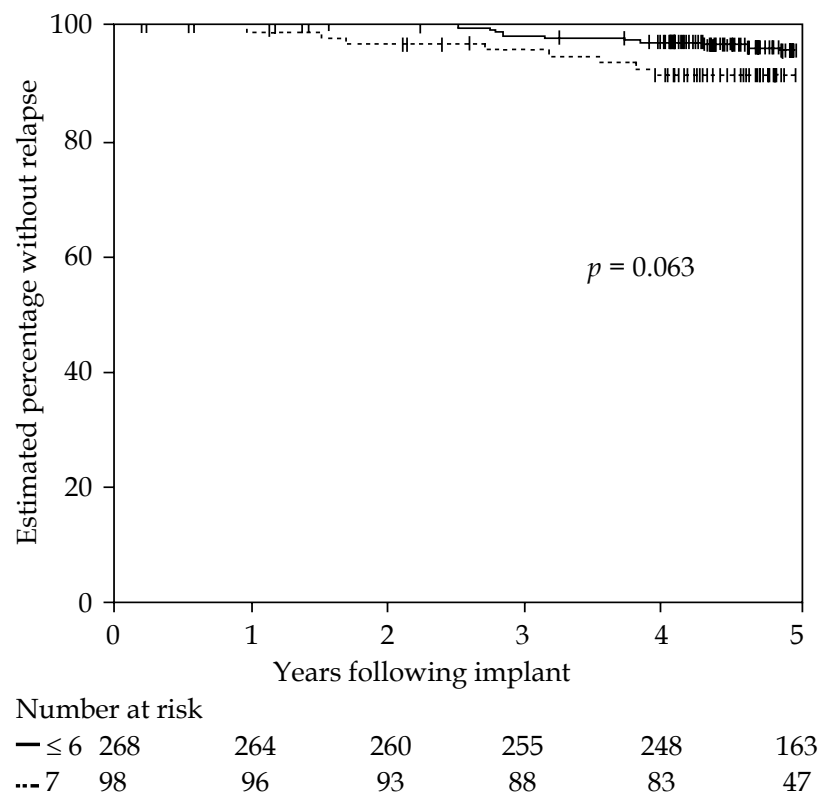

Fig. 1. Time to relapse by Gleason score such as pre-implant PSA had a significant effect on time to relapse (all $p>0.1$ ). In the 18 patients (5\%) with PSA relapse, seven patients have developed local recurrence only, whilst two patients experienced either concurrent nodal relapse or distant metastases. Only one patient developed distant metastases alone. To date, no patient has clinically significant local disease.

\section{PSA bounce}

A PSA bounce was observed in 137 patients (37\%). The median time to developing a bounce was 1.47 years (IQR: 0.34-3.31 years), and the magnitude of the PSA rise during the bounce period was a median of $0.6 \mathrm{ng} / \mathrm{ml}$ (IQR: 0.2-6.1 ng/ml). A significantly higher chance of developing a bounce was observed in younger (i.e. $<59$ years of age) rather than older patients $(p<0.0001)$. Other prognostic factors such as $\mathrm{T}$ stage, prior hormone therapy, and prostate volume had no significant effect on whether the patient had a PSA bounce (all $p>0.4$ ).

\section{Overall survival}

The overall survival at 5 years according to Kaplan-Meier estimate was 96\% (95\% CI: 93-98\%) for all patients, and was not significant between low 97\% (95\% CI: $94-98 \%$ ) and intermediate $94 \%$ (95\% CI: 88-98\%) risk groups $(p=0.41)$. Univariate regression revealed no statistical association for clinical T stage, Gleason scores, by risk group, or prostate volume. There were 14 deaths in total and none were attributed to prostate cancer. Of the 14 deaths, seven deaths were due to other cancers, and seven deaths were due to other causes. The other cancers were: colorectal $(n=2)$ and one each of TCC bladder, non-squamous cell lung, pancreas, leukemia, and lymphoma. The other causes were: cerebrovascular accident $(n=2)$ and one each of acute myocardial infarct, deep vein thrombosis, and ensuing pulmonary embolism, ischemic heart disease, pneumonia, and unknown cause (Figure 2).

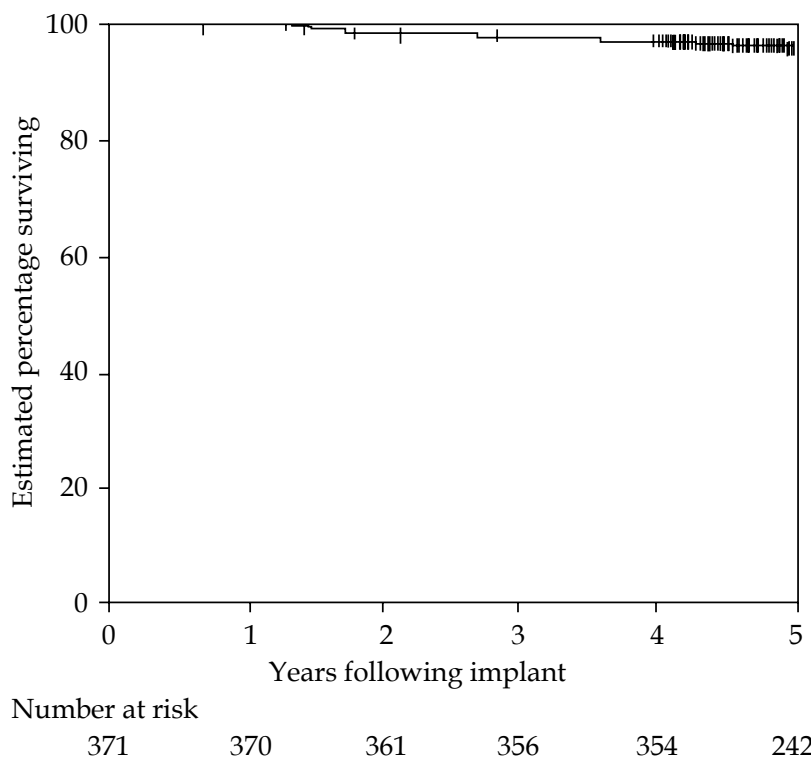

Fig. 2. Overall survival for all patients 


\section{Late toxicities}

Only $6 \%$ of patients experienced grade 3 urinary retention symptoms. Patients who underwent a pre-implant TURP were at a higher risk of developing subsequent grade 2 urinary retention toxicities $(p=0.008)$ however, no difference between TURP and non-TURP cohorts was seen in grade 3 urinary retention toxicities. Urinary incontinence requiring pads occurred in three patients $(<1 \%)$, whilst grade 3 hematuria toxicities occurred in $2 \%$ of patients, more common in patients who underwent a pre-implant TURP $(p<0.001)$. No patients including those who had a pre-implant limited TURP developed urethral necrosis or recto-prostatic fistulas. For more detail, see Table 3 .

\section{Discussion}

The aim of this prospective case series was to report on the safety and effectiveness of LDR brachytherapy for patients with prostate cancer. The results showed our 5-year OS and RFS rates to be $96 \%$ and $95 \%$ respectively. More so, $80 \%$ of patients had reached their PSA nadir at 5 -years after implant and only a handful of patients $(5 \%)$ relapsed within the post-implant follow-up period. In addition, we noted the observed difference between the planned and delivered $D_{90}$ values in our population. At 3-months, 35\% of patients had grade 1 toxicities (i.e. hematuria), whilst a small sample of patients $(2 \%)$ developed grade 3 toxicities. A handful of grade 3 late toxicities were observed with urinary retention $(6 \%)$, hematuria $(2 \%)$, and urinary incontinence $(<1 \%)$. All other events varied from grade 1 to 2 .

Our results were consistent with other international studies, which showed very high local control rates postLDR brachytherapy. In the study by Peacock et al. [3], 822 patients were treated with LDR brachytherapy alone between 2003-2013. At 5- and 10-years, the biochemical freedom from relapse was $95 \%$ and $87 \%$, respectively. In the smaller study by Rea et al. [7], 110 patients ( $n=71$ low-risk; $n=33$ intermediate-risk) underwent LDR brachytherapy, in which the authors observed the biochemical freedom from relapse rate to be $98.5 \%$ in the low-risk group and $81.8 \%$ in the intermediate group. In the study by Logghe et al. [17], 274 patients with organ-confined prostate cancer were treated with LDR brachytherapy. Follow-up 5-year data revealed disease-specific survival and overall survival to be $98.5 \%$ and $93.5 \%$. Similar to our study numbers, 20 patients developed either local or systemic disease.

Our results were also consistent with other studies in our region. In the study by Millar et al. [18], the authors reported on 582 patients who were treated with permanent implant brachytherapy monotherapy for prostate cancer. At 5 - and 8 -years, OS was $97 \%$ and $94 \%$. In a second study by Wilson et al. [19], the authors reported long-term PSA and toxicity outcomes for patients with localized prostate cancer treated with LDR brachytherapy. More specifically, the 10-year biochemical disease-free survival for the entire cohort was $89 \%$, with Kaplan Meier estimates by pre-treatment risk group to be $96 \%$ and $83 \%$ for low- and intermediate-risk, respectively.

After brachytherapy, the PSA bounce was observed in $37 \%$ of patients almost 18 months after commencement of treatment. In most instances, this 'spike' effect was seen in our cohort of younger patients (i.e. $<59$ years). Similarly, Kindts et al. [20] noted 70 patients (36\%) developing a bounce at 18 months, with a mean magnitude of the PSA rise of $0.67 \mathrm{ng} / \mathrm{ml}$. In addition, the authors observed that younger age and lower treatment activity per volume were significant factors associated with a higher chance of developing a bounce. Both findings reflected recent appropriate criteria for brachytherapy, with consensus views on management strategies as described by the American College of Radiology [21]. In particular, it was reported that PSA bounces were observed in up to $40-50 \%$ of all hormone-naïve patients, mostly in those patients that were younger, and often 12-30 months after treatment.

In our cohort of 371 patients, which included 102 patients with a prior history of TURP, we were able to demonstrate high local control rates post-brachytherapy. In the study by Prada et al. [6], 57 patients with clinically localized prostate cancer that underwent LDR brachytherapy were followed up for a period of 10 years. Follow-up 5-year data revealed biochemical control, tumor-free survival, and OS were $94 \%, 96 \%$, and $88 \%$, respectively. In studies by Brousil et al. [22] and Salembier et al. [23], the authors observed that prostate brachytherapy relative to the TURP did not hinder proper dosimetric outcomes or result in significant toxicities. In our study, almost one-third of our population had a TURP prior to brachytherapy, which did not hinder dosimetric outcomes. However, the risk of subsequent grade 2 urinary retention was increased. This was usually managed with short-term intermittent self-catheterization. No patients who underwent a pre-implant TURP developed urethral necrosis or recto-prostatic fistulas.

Table 3. Late toxicities

\begin{tabular}{lccc} 
Late toxicities & Grade & $n$ & $\%$ \\
\hline Proctitis & 1 & 20 & 5 \\
& 2 & 1 & $<1$ \\
\hline Urinary incontinence & 1 & 1 & $<1$ \\
& 2 & 2 & $<1$ \\
& 3 & 1 & $<1$ \\
\hline Urinary retention (stricture) & 0 & 226 & 86 \\
& 1 & 8 & 3 \\
No prior TURP & 2 & 13 & 5 \\
\hline Urinary retention (stricture) & 3 & 15 & 6 \\
& 0 & 75 & 74 \\
Prior TURP & 1 & 2 & 2 \\
\hline Hematuria & 2 & 18 & 18 \\
& 3 & 7 & 7 \\
No prior TURP & 0 & 258 & 98 \\
\hline Hematuria & 1 & 3 & 1 \\
Prior TURP & 3 & 1 & $<1$ \\
Data presented as number (percentage) & 3 & 7 & 88 \\
TURP - transurethral resection of the prostate & & 7 \\
& 1 & 5 &
\end{tabular}


We were also mindful of the current debate surrounding the relationship between dosimetric criteria and relapse-free survival and/or survival [24] given that our median $\mathrm{D}_{90}$ post-implant was 144 Gy (range, 64-215). However, we observed that other studies reported no statistically significant effect on biochemical disease-free survival or survival when stratified into two (i.e. $\mathrm{D}_{90}$ $<140 \mathrm{~Gy}$ and $>140 \mathrm{~Gy}$ ) or three groups (i.e. $\mathrm{D}_{90} \leq 160 \mathrm{~Gy}$; 160-180 Gy; $\geq 180$ Gy). Conversely, Rasmusson et al. [4] found a significant relationship between $\mathrm{D}_{90}$ and biochemical failure-free survival, with 167 Gy considered as the tipping point. However, in our present case series, we did not stratify for arbitrary dose levels.

Given that brachytherapy may cause toxicities as a result of a combination of local trauma and radiation, we were not surprised to confirm examples of long-term GU toxicities (i.e. urinary retention) in $6 \%$ of our patients. The rates of late grade $3 \mathrm{GU}$ toxicities found in our analysis were similar to those published in other studies that included prostate volumes > 50 cc [25]. However, Millar et al. [18] noted Grade 3 complications (i.e. mostly urinary retention) occurring in only $2.9 \%$ of patients. Regardless of these minor rates differences, our findings suggested that implantation of larger glands with seeds was safe in this regard.

\section{Study strengths and limitations}

A key strength of our study was that we collected data in a prospective fashion from 371 patients attending a specialist radiation oncology center in Melbourne, Australia. Secondly, feasibility of implant was robustly assessed prior to implant in our TURP cohort, almost one-third of our population. Prior TURP did not hinder dosimetric outcomes or result in significant toxicities. Thirdly, we adhered to the parameters established by the American College of Radiology [2] and the American Brachytherapy Society [26] for transperineal permanent brachytherapy of prostate cancer. Lastly, we reported minimal long-term grade 3 adverse events throughout the 5-year follow-up period. This study also had a series of limitations. In particular, our findings were restricted to a non-randomized and non-blinded approach, which meant it was prone to methodological shortcomings. However, by using this case series design, we demonstrated the overall safety of brachytherapy in patients with prostate cancer. Secondly, our long-term follow-up of patients reported on disease-specific outcomes only. While important, we may have also missed out on understanding the impact of disease on general and disease-specific health-related quality of life.

\section{Conclusions}

The results of our prospective case series are in parallel with regionally-based published research that has now provided clinicians with long-term data about the benefit of LDR brachytherapy for patients with clinically localized, low- to intermediate-risk prostate cancer. In particular, these encouraging findings would suggest brachytherapy to be as effective as other radiation and surgical treatment modalities. The observed low to moderate long-term com- plication rate would suggest that the regime offered in this study was feasible and well tolerated. Our results indicate that a prior limited TURP is not a contraindication for LDR brachytherapy, in which the implant is technically feasible. However, the risk of urinary retention is increased. Further studies using multi-institutional centers, larger sample size, and disease-specific and overall health outcome instruments are needed to clarify the direct impact of brachytherapy on prostate cancer.

\section{Disclosure}

The authors report no conflict of interest.

\section{References}

1. Major T, Polgár C, Jorgo $\mathrm{K}$ et al. Dosimetric comparison between treatment plans of patients treated with low-doserate vs. high-dose-rate interstitial prostate brachytherapy as monotherapy: Initial findings of a randomized clinical trial. Brachytherapy 2017; 16: 608-615.

2. Dickinson PD, Malik J, Mandall P et al. Five-year outcomes after iodine-125 seed brachytherapy for low-risk prostate cancer at three cancer centres in the UK. BJU Int 2014; 113: 748-753.

3. Peacock M, Martell K, Taggar et al. Institutional long-term outcomes at the first Canadian center performing intraoperatively planned low-dose-rate brachytherapy alone in lowand intermediate-risk prostate cancer. Brachytherapy 2017; 16 : 822-830.

4. Rasmusson E, Gunnlaugsson A, Kjellén E et al. Low-dose rate brachytherapy with I-125 seeds has an excellent 5-year outcome with few side effects in patients with low-risk prostate cancer. Acta Oncol 2016; 55: 1016-1021.

5. Bolla, M, Verry C, Giraud JY et al. Results of a cohort of 200 hormone-naïve consecutive patients with prostate cancer treated with iodine 125 permanent interstitial brachytherapy by the same multidisciplinary team. Cancer Radiother 2014; 18: 643-648.

6. Prada PJ, Anchuelo J, Blanco AG et al. Low-dose-rate brachytherapy for patients with transurethral resection before implantation in prostate cancer. Longterm results. Int Braz J Urol 2016; 42: 47-52.

7. Rea A, Rogers P, Jones A. Ten year outcomes of real time " $4 \mathrm{D}$ " brachytherapy in prostates up to $100 \mathrm{cc}$. Eur Urol Suppl 2017; 16: e1408.

8. Stish BJ, Davis BJ, Mynderse LA et al. Brachytherapy in the management of prostate cancer. Surg Oncol Clin N Am 2017; 26: 491-513.

9. Rodrigues G, Yao X, Loblaw DA et al. Low-dose rate brachytherapy for patients with low- or intermediate risk prostate cancer: A systematic review. Can Urol Assoc J 2013; 7: 463-470.

10. Ng M, Brown E, Williams A et al. Fiducial markers and spacers in prostate radiotherapy: current applications. BJU Int 2014; 113 Suppl 2: 13-20.

11. Cihoric N, Tsikkinis A, Miguelez CG et al. Portfolio of prospective clinical trials including brachytherapy: an analysis of the ClinicalTrials.gov database. Radiat Oncol 2016; 11: 48.

12. Orio PF 3rd, Nguyen PL, Buzurovic I et al. Prostate brachytherapy case volumes by academic and nonacademic practices: implications for future residency training. Int J Radiat Oncol Biol Phys 2016; 96: 624-628.

13. D'Amico AV, Whittington R, Malkowicz SB et al. Biochemical outcome after radical prostatectomy, external beam radiation therapy, or interstitial radiation therapy for clinically localized prostate cancer. JAMA 1998; 280: 969-974. 
14. Rivard MJ, Coursey BM, DeWerd LA et al. Update of AAPM Task Group No. 43 Report: A revised AAPM protocol for brachytherapy dose calculations. Med Phys 2004; 31: 633-674.

15. Thompson A, Keyes M, Pickles T et al. Evaluating the Phoenix definition of biochemical failure after 125I prostate brachytherapy: can PSA kinetics distinguish PSA failures from PSA bounces? Int J Radiat Oncol Biol Phys 2010; 78: 415-421.

16. Mazeron R, Bajard A, Montbarbon X et al. Permanent 125I-seed prostate brachytherapy: early prostate specific antigen value as a predictor of PSA bounce occurrence. Radiat Oncol 2012; 7: 46

17. Logghe $\mathrm{P}$, Verlinde $\mathrm{R}$, Bouttens $\mathrm{F}$ et al. Long term outcome and side effects in patients receiving low-dose I125 brachytherapy: a retrospective analysis. Int Braz J Urol 2016; 42: 906-917.

18. Millar JL, Matheson B, Hindson B et al. Disease-control outcomes of permanent Iodine-125 seed implantation for prostate cancer at a single Australian institution. Brachytherapy 2013; 12 (Suppl 1): S13-14.

19. Wilson C, Waterhouse D, Lane SE et al. Ten-year outcomes using low dose rate brachytherapy for localised prostate cancer: An update to the first Australian experience. J Med Imaging Radiat Oncol 2016; 60: 531-538.

20. Kindts I, Stellamans K, Billiet I et al. ${ }^{125} \mathrm{I}$ brachytherapy in younger prostate cancer patients: Outcomes in low- and intermediate-risk disease. Strahlenther Onkol 2017; 193: 707-713.

21. Davis BJ, Taira AV, Nguyen PL et al. ACR appropriateness criteria: Permanent source brachytherapy for prostate cancer. Brachytherapy 2017; 16: 266-276.

22. Brousil P, Hussain M, Lynch et al. Modified transurethral resection of the prostate (TURP) for men with moderate lower urinary tract symptoms (LUTS) before brachytherapy is safe and feasible. BJU Int 2015; 115: 580-586.

23. Salembier C, Rijnders A, Henry A et al. Prospective multi-center dosimetry study of low-dose Iodine-125 prostate brachytherapy performed after transurethral resection. J Contemp Brachytherapy 2013; 5: 63-69.

24. Cosset JM, Flam T, Belin L et al. Long-term results of permanent implant prostate cancer brachytherapy: A single-institution study of 675 patients treated between 1999 and 2003. Cancer Radiother 2016; 20: 261-267.

25. Pham YD, Kittel JA, Reddy CA et al. Outcomes for prostate glands $>60 \mathrm{cc}$ treated with low-dose-rate brachytherapy. Brachytherapy 2016; 15: 163-168.

26. Bittner NH, Orio PF 3rd, Merrick GS et al. The American College of Radiology and the American Brachytherapy Society practice parameters for transperineal permanent brachytherapy of prostate cancer. Brachytherapy 2017; 16: 59-56. 